Daniel Weidner · Sigrid Weigel (Hrsg.)

Benjamin-Studien 2 

Daniel Weidner · Sigrid Weigel (Hrsg.)

Benjamin-Studien 2

Wilhelm Fink 
Bibliografische Information der Deutschen Nationalbibliothek

Die Deutsche Nationalbibliothek verzeichnet diese Publikation in der Deutschen Nationalbibliografie; detaillierte bibliografische Daten sind im Internet über http://dnb.d-nb.de abrufbar.

Alle Rechte, auch die des auszugsweisen Nachdrucks, der fotomechanischen Wiedergabe und der Übersetzung, vorbehalten. Dies betrifft auch die Vervielfältigung und Übertragung einzelner Textabschnitte, Zeichnungen oder Bilder durch alle Verfahren wie Speicherung und Übertragung auf Papier, Transparente, Filme, Bänder, Platten und andere Medien, soweit es nicht $\$ \$ 53$ und 54 UrhG ausdrücklich gestatten.

(C) 2011 Wilhelm Fink Verlag, München

(Wilhelm Fink GmbH \& Co. Verlags-KG, Jühenplatz 1, D-33098 Paderborn)

Internet: www.fink.de

Die Drucklegung dieses Werkes wurde unterstützt mit den Mitteln des Bundesministeriums für Bildung und Forschung unter dem Förderkennzeichen 1UG0712.

Lektorat: Bettina Moll, Berlin

Satz: Tilo Lothar Rölleke, Berlin

Einbandgestaltung: Evelyn Ziegler, München

Printed in Germany

Herstellung: Ferdinand Schöningh GmbH \& Co. KG, Paderborn

ISBN 978-3-7705-5071-5 


\section{DANIEL WEIDNER}

\section{Fort-, Über-, Nachleben $\mathrm{Zu}$ einer Denkfigur bei Benjamin}

Durch Benjamins Texte zieht sich eine deutlich erkennbare Spur eines Versuches, Geschichte als Zusammenhang des `Lebens` und `Nachlebens` zu denken. In Die Aufgabe des Übersetzers spricht er nicht nur vom »Überleben" des Originals in der Übersetzung, sondern formuliert auch allgemeiner und »in völlig unmetaphorischer Sachlichkeit« den "Gedanke[n] vom Leben und Fortleben der Kunstwerke« (GS IV, 11). Diese Formulierungen werden wieder aufgegriffen in den Überlegungen über die Vor- und Nachgeschichte der Kunstwerke im Trauerspielbuch, die hier an zentraler Stelle als »Leben der Werke und Formen« bzw. als »natürliches Leben« charakterisiert werden (GS I, 227). In den Fragmenten zur Literaturkritik wird als auszuarbeitendes Projekt die »Lehre vom Fortleben der Werke« erwähnt (GS VI, 174), im Aufsatz über Eduard Fuchs wird geschichtliches Verstehen insgesamt »als Nachleben des Verstandenen auf[gefasst], dessen Pulse bis in die Gegenwart spürbar sind (GS II, 468) und im Passagenwerk heißt es schließlich: "Geschichtliches ,Verstehen ist grundsätzlich als ein Nachleben des Verstandnen zu fassen und daher ist dasjenige was in der Analyse des `Nachlebens der Werke`, des 'Ruhmes erkannt wurde, als die Grundlage der Geschichte überhaupt zu betrachten« (GS V, 574 f.).

Diese Spur ist gegenwärtig umso interessanter, als die Figur des Nachlebens heute zu den zentralen Begriffen einer Kulturwissenschaft gehört, die sich am Paradigma des kulturellen Gedächtnisses orientiert. Dabei wird auch explizit auf Diskurse aus der ersten Hälfte des 20. Jahrhunderts verwiesen, insbesondere auf Aby Warburgs Untersuchung des 'Nachlebens` der Antike, in dem etwa Georges DidiHuberman einen alternativen Entwurf einer Kunstgeschichte angelegt sieht:

An die Stelle des natürlichen Modells der Kreisläufe `Leben und Tod`, ‘Größe und Verfalk setzte Warburg ein entschieden nichtnatürliches, symbolisches Modell, ein kulturelles Modell der Geschichte, in dem die Zeit nicht auf biomorphe Stadien projiziert wird, sondern ihren Ausdruck in Schichten, hybriden Blöcken, Rhizomen, spezifischen Komplexitäten, oft unerwarteten Rückwendungen und stets verfehlten Zielen findet. ${ }^{1}$

1 Georges Didi-Huberman: Das Nachleben der Bilder. Kunstgeschichte und Phantomzeit nach Aby Warburg, Berlin (Suhrkamp) 2010, S. 30. Didi-Huberman zeichnet auch nach, wie Warburgs Anliegen bei seinen Schülern auf die Frage nach Quellen, Einflüssen oder gar Überresten reduziert worden ist, vgl. ebd. S. $102 \mathrm{ff}$. Zum Nachleben allgemein vgl. programmatisch 
Die gespenstische Zeitlichkeit einer solchen Geschichte hat man auch Benjamins Texten immer wieder zu Recht zugeschrieben; sie gilt nicht zuletzt auch für deren eigene Geschichte, insofern Benjamins ,Werk` heute als nachträgliche Konstruktion erkennbar ist. ${ }^{2}$ Allerdings werden seine eigenen Überlegungen zum `Nachleben` dabei meist lediglich zitiert, aber nur selten als eigenes Projekt wahrgenommen. ${ }^{3}$ In den zahllosen Untersuchungen zu Benjamins Gedächtnisund Geschichtskonzeption spielt das `Nachleben` nur eine untergeordnete Rolle; wo es eigens in den Blick genommen wird, wird es durch andere Konzepte wie `Zitatı oder `Erbe perspektiviert; insbesondere wird das naheliegende Verhältnis des `Nachlebens` zum Leben nur am Rande berührt, obwohl die Rede vom "Leben und Fortleben der Kunstwerke" gerade diesen Zusammenhang zu betonen scheint. ${ }^{4}$ Wenn man aber diese Beziehung außer Acht lässt, erscheint 'Nachleben` fast notwendig als relativ beliebige Metapher, die man dann selbst

Martin Treml/Sigrid Weigel: ")Nachleben` in den Kulturwissenschaften - Gegenstand und Methode", in: Trajekte Extra, Berlin 2006, S. 13-16 sowie Martin Treml/Daniel Weidner (Hg.): Nachleben der Religionen. Kulturwissenschaftliche Untersuchungen zur Dialektik der Säkularisierung, München (Fink) 2007. Zum Nachleben in der Geschichte vgl. auch Ulrich Raulffs "Gespenstergeschichte«, in ders.: Kreis ohne Meister. Stefan Georges Nachleben, München (C. H. Beck) 2009, S. 22.

2 Vgl. unter vielem den Band Gerhard Richter (Hg.): Benjamin's Ghosts. Interventions in Contemporary Literary and Cultural Theory, Stanford 2002. Zur nachträglichen Werkkonstruktion vgl. Detlev Schöttker: Konstruktiver Fragmentarismus. Form und Rezeption der Schriften Walter Benjamins, Frankfurt a. M. (Suhrkamp) 1999.

3 Im von Burkhard Lindner herausgegebenen Benjamin-Handbuch (Stuttgart 2006) findet sich kein Registereintrag `Nachleben`, zur `Nachgeschichte finden sich nur zwei wenig einschlägige Verweise, ,Fortleben verweist immerhin auf Benjamins Erörterung über Briefe als ZZeugnisser. Noch auffälliger ist diese Lücke, wenn in dem Band Archiv-Zitat - Nachleben. Die Medien bei Walter Benjamin und das Medium Benjamin (hg. v. Amália Kerekes/Nicolas Pethes/Peter Plener, Frankfurt a. M. [Lang] 2005) das titelgebende «Nachleben einem der Beiträge noch in der Einleitung zum Gegenstand wird.

4 Bettine Menke untersucht das Nachleben lektüretheoretisch vom Zitat her und betont dessen zersetzende Gestalt (»Das Nach-Leben im Zitat. Benjamins Gedächtnis der Texte«, in: Anselm Haverkamp/Renate Lachmann (Hg.): Gedächtniskunst: Raum - Bild - Schrift, Frankfurt a. M. [Suhrkamp] 1991, S. 74-110). Stefan Willer ordnet die Rede vom Nachleben in die Debatte über das kulturelle Erbe ein: "Das Destruktive im Erbe ist schlicht und einfach [...] das Faktum des Todes, an das jeder Erbfall, auch der kulturelle, zwangsläufig gebunden ist. Im Nachleben, wie es Benjamin denkt, ist die Zäsur des Ablebens sehr deutlich hervorzuheben" (»Nachleben des Verstandenen،. Walter Benjamin und das Erbe des historischen Materialismus", in: Text + Kritik. Walter Benjamin 31/32 [2009], S. 88-96, hier S. 93). Anders als diese beiden Aufsätze setzt sich Samuel Weber auch mit den Passagen aus dem Übersetzer-Aufsatz auseinander, in denen Benjamin eine Geschichte des Sterblichen entwickele, "a history that is mindful of mortalitiy, a history that does not try to overcome or transfigure the finitude" („Translatability II: Afterlife«, in: Samuel Weber: Benjamin's Abilities, Cambridge/Mass. [Harvard University Press] 2008, S. 79-94, hier S. 67). Zu Benjamins Überlegungen zum Fortleben als Entwurf einer Rezeptionsgeschichte, vgl. Schöttker: Konstruktiver Fragmentarismus (Anm. 2), S. $94 \mathrm{ff.}$ 
wiederum schmückend zitieren kann, ohne die sie eigentlich fundierende Problematik nachzuvollziehen. Dabei lässt sich gerade am Schritt vom `Leben zum `Nachleben` nicht nur zeigen, auf welche zeitgenössischen Diskurse Benjamin Bezug nimmt und wie er mit ihnen umgeht; dieser Schritt macht auch zuallererst erkennbar, worin die eigentliche Prägnanz der Benjamin'schen Rede vom 'Nachleben` besteht und welchen Status sie hat: Es handelt sich weder um einen Terminus noch um einen beliebigen Ausdruck, sondern um eine Denkfigur, die auf rhetorischen Operationen wie der erwähnten Verschiebung und deren präzisem Vollzug in Benjamins Texten beruht. Im Folgenden soll daher zunächst (1) an einem konkreten Einzelfall die zeitgenössische Bedeutung von Nachleben und (2) der diskursive Hintergrund der 'Lebensphilosophie werden, dann (3) die Bedeutung des ,Lebens` im Übersetzeraufsatz untersucht werden, um darauf aufbauend (4) die argumentative Verschiebung vom \Leben` zum ,Überleben`nachzuzeichnen sowie (5) die religiöse Dimension des 'Nachlebens` und (6) die von ihm implizierte zugrundliegende Zeitlichkeit zu erörtern.

1.

Die Rede vom 'Nachleben philologie eingeführter Titel für Fragen der Rezeptionsgeschichte, ohne einen terminologischen Status zu haben. ${ }^{5}$ Auch Aby Warburg entwickelt niemals eine geschlossene Theorie des Nachlebens und verwendet den Ausdruck in seinem Werk überhaupt eher beiläufig. ${ }^{6}$ Gerade NNachleben der Antike` steht zunächst meist für eine diffuse Verbindung eines historischen Interesses mit einer kulturpolitischen Agenda. Exemplarisch dafür mag Otto Immischs Broschüre Das Nachleben der Antike stehen, die 1919 als Heft 1 der ebenfalls von Immisch herausgegebenen Reihe »Das Erbe der Alten. Schriften über Wesen und Wirkung der Antike«, Neue Folge, erscheint, also durchaus in einer programmatischen

5 Zum Begriffsgebrauch in der Altertumswissenschaft vgl. den Literaturbericht von Richard Newald, der eine Fülle von Titeln unter diesem (offensichtlich durchaus gebräuchlichen) Titel bespricht und zusammenfasst: "Die Antike ist geistiges Erbgut von ewiger Frische, nicht tote Masse, nicht Gesetz, sondern ein Füllhorn der Weisheit und Kunst, aus dem sich jede Zeit und jeder selbständige Denker das Wahlverwandte sucht" (Richard Newald: »Nachleben der Antike (1920-1929)«, in: Jahresberichte über die Fortschritte der klassischen Altertumswissenschaft, Bd. 232 [1931], 3. Abt., S. 1-110, hier S. 2). Zu ähnlichen Untersuchungen bereits im 19. Jahrhundert vgl. Didi-Huberman: Das Nachleben der Bilder (Anm. 1), S. 93 ff.

6 Erst durch die Lemmatisierung seiner 1932 etablierten Gesammelten Schriften verband sich der Ausdruck mit einem Forschungsprogramm, vgl. Martin Treml: „Warburgs Nachleben", in: ders./Weidner (Hg.): Nachleben der Religionen (Anm. 1), S. 25-40, hier auch weiteres zum Gebrauch ähnlicher Ausdrücke bei Darwin und Tylor. 
Position. Immisch, Altphilologe in Freiburg und Mitglied des Gymnasialverbandes zum Erhalt des humanistischen Gymnasiums, betont die dauernde Bedeutung der kulturellen Vergangenheit gerade in einer Zeit der Krise: „Die alten Sterne leuchten wieder, auch uns. Wir sehen auch auf diesem Wege, mit der Einmaligkeit der geschichtlichen Erscheinung stimmt es nicht, es gibt ein Erneuern, ein Wiederaufleben, das viel mehr ist als bloße geschichtliche Rekonstruktion. " ${ }^{7}$ Die Antike sei immer noch lebendig, denn es gäbe auch in der Welt des Geistes ein Gesetz der Erhaltung der Energie: "Auch das Geistige, ist es erst einmal geformt hervorgetreten, bleibt 'geprägte Form, die lebend sich entwickeltı. Und weil lebend, so auch irgendwie wirkend. Diese Wirkung lässt sich nun freilich (im verwandlungsreichen Fortzeugungsprozess der Natur ist es aber nicht anders) von dem ursprünglichen Gebilde los, passt sich neuen Voraussetzungen an, lebt und webt so weiter zu eigenem Recht und erzeugt immer weitere Nachwirkungen mit der gleichen Umbildungsfähigkeit und Selbstständigkeit. ${ }^{8}$ Daher gehe das Historische auch nicht in seinem von der historischen Wissenschaft festzustellenden Ursprung auf, sondern stelle eine »lange Reihe von Kulturwirkungen« dar, die doch insgesamt ein Ganzes bilden: "Alles überhaupt, was lebte und wirkte, lebt und wirkt auch weiter." ${ }^{9}$ Immisch will daher in diesem Sinne die Allgegenwart von Wirkungen der Antike aufzeigen, um sowohl die Gegenwart besser zu verstehen als auch die Antike "von der Starrheit des Ausgelebten und Gewesenen« zu befreien: »sie erscheint als ein Teil unseres eigenen Lebensprozesses und darin liegt, dass sie selber noch flüssig und wandelbar wie alles Lebendige auch für die Zukunft noch neue Wirkungen und Aneignungsmöglichkeiten entfalten kann. ${ }^{10}$

Immischs Verwendung des Begriffs dürfte relativ typisch sein. Die Rede einer lebendigen ‘ Antike rekurriert zunächst auf den 'Kampfbegriffı des Lebens, der sich gegen alles Tote und Abgelebte richtet. Die Rede vom Nachleben impliziert aber noch mehr, sie verbindet die Behauptung einer (vermittelten) Wirkungsgeschichte, in der die Geschichte lebendig geblieben ist, mit einer (unmittelbaren) Relevanz für die Gegenwart, in der die Antike für uns noch lebensbestimmend

7 Otto Immisch: Das Nachleben der Antike, Leipzig (Dieterich) 1919, S. 15 f. Ich danke Ernst Müller für den Hinweis auf diese Broschüre. Auch bei Warburg finden sich Notizen zum `Nachleben` im Umkreis des Kampfs um das deutsche Gymnasium, vgl. Treml: „Warburgs Nachleben« (Anm. 6), S. 39.

8 Immisch: Das Nachleben der Antike (Anm. 7), S. 16.

9 Ebd., S. 17 f. Immisch zieht hier auch eine interessante Parallele zum Christentum, insofern auch Jesus historisch im Kontext seiner Zeit verstanden werde, »doch gehört untrennbar zu ihm auch das Evangelium und die Kirche, sein ganzes stets gewandeltes, aber noch immer wandelbares und bis auf den heutigen Tag unerschöpftes Nachleben - wahrlich keine `Einmaligkeit‘, sondern als ganzes ein zusammenhängender Energieumsatz« (ebd., 16).

10 Ebd., S. 19. Der Text enthält auch eine ganze Reihe anderer Metaphern des Erbes, etwa "Gold bleibt Gold, auch wenn es immer wieder neugemünzt werden muß« (ebd. S. 61 f.). 
sein kann. Immischs Appell wird also gerade plausibel, weil `Nachleben der Antiker offen lässt, ob der Genitiv subjektiv oder objektiv gemeint ist: ob die Antike weiterlebt oder ob wir der Antike nachleben, sie nacherleben sollen, wie es der Neuhumanismus wieder und wieder fordert. Das 'Leben gangenheit und Zukunft, es bezeichnet als Kollektivsingular >die Geschichter als ganze und als je einzelnes Leben die individuelle Einheit eines Werkes oder Werkzusammenhanges. All diese semantischen Reserven können auch in die Rede vom `Nachleben` einwandern und prädestinieren den Ausdruck dazu, zu einer Figur zu werden. ${ }^{11}$

2.

Immisch bezog sich auf Goethes Urworte, Orphisch (»keine Zeit und keine Macht zerstückelt / geprägte Form, die lebend sich entwickelt») und auch dieser Rekurs ist typisch, weil die Zeitgenossen die verschiedenen Dimensionen der Lebenssemantik immer wieder an Goethe festmachten: Dieser verkörperte den Zusammenhang einer morphologischen Theorie des Lebendigen mit einer ganzheitlichen Anschauung des eigenen Lebens und der exemplarischen Vergegenwärtigung der Geschichte im eigenen Leben. Für Wilhelm Dilthey etwa lässt sich Geschichte nur als Zusammenhang denken, der stets auf das Individuum und sein Verstehen bezogen ist und als "Zusammenhang des Lebens" beschrieben werden kann - eine Idee des Lebens, die weniger von der Biologie als von der Historie und dem historischen Verstehen her zu denken ist. Dieses Leben wird daher nach Dilthey paradigmatisch erkennbar an der Selbstbiographie und dort wiederum paradigmatisch an Goethes Dichtung und Wahrheit: "Der Sinn des Lebens liegt in der Gestaltung, in der Entwicklung; von hier aus bestimmt sich die Bedeutung der Lebensmomente auf eine eigene Weise; sie ist zugleich erlebter Eigenwert des Momentes und dessen wirkende Kraft. $\aleph^{12}$ Die zu begründenden Geisteswissenschaften könnten also mit gleichem Recht 'Lebenswissenschaften genannt werden, wobei die Vorstellung eines >organischen Zusammenhangs des Lebens mit der des deutenden Erlebens dieses Zusammenhangs verbunden wird; daher wird die Objektivierung des Erlebnisses in der Dichtung einerseits, die

11 Zur Semantik von Leben vgl. auch Hans Blumenberg: Theorie der Lebenswelt, Berlin (Suhrkamp) 2010. S. 9-24.

12 Wilhelm Dilthey: Gesammelte Schriften, Bd. 7: Der Aufbau der geschichtlichen Welt in den Geisteswissenschaften, Göttingen (Vandenhoeck \& Ruprecht) 1992, S. 199. "Jedes Leben hat seinen eigenen Sinn. [...] Dieser Sinn des individuellen Daseins ist ganz singular, dem Erkennen unauflösbar, und er repräsentiert doch in seiner Art, wie eine Monade von Leibniz, das geschichtliche Universum» (ebd.). Über den "Zusammenhang des Lebens« vgl. ebd. S. $196 \mathrm{ff}$. 
organische Entwicklung dieser Dichtung andererseits, später zu den Hauptinteressen dieser Wissenschaft gehören. ${ }^{13}$ Allerdings wird diese Semantik des Lebens` auch weiterentwickelt und gerät damit auch in eine latente Krise, besonders beim späten Georg Simmel, den Benjamin 1912/1913 an der Universität gehört hat und dessen Texte er bekanntlich immer wieder erwähnt. Auf der einen Seite spricht Simmel dem `Leben zeittypisch eine universelle Integrations- und Vermittlungsfunktion zu, in der das Lebendige immer und stets alle festen Formen auflöst. Auf der anderen Seite entdeckt er in dieser Vermittlung ein konträres Element, welches das Leben untergräbt, und zwar gerade, indem die Semantik des `Lebens` entwickelt und universalisiert wird.

Auch Simmel entwirft Geschichte als Lebensprozess: In Der Konflikt der modernen Kultur (1918) heißt es zunächst, die Differenz zwischen je besonderen kulturellen Formen und dem immer wandelbar-strömenden Leben habe zur Folge, "daß die Kultur eine Geschichte hat. " ${ }^{14}$ In Lebensanschauung (1918) wird diese Geschichte nun aber in das Leben selbst hinein verlegt: Das »Hinausgreifen des aktuellen Lebens in dasjenige, was nicht seine Aktualität ist [...] ist also nichts, was zum Leben erst hinzukäme, sondern dieses, wie es in Wachstum und Zeugung und in den geistigen Prozessen sich vollzieht, ist das Wesen des Lebens selbst «. ${ }^{15}$ Das Leben ist immer über sich selbst hinaus, und das drückt sich nun auch in seiner Beschreibung, in der Rhetorik des Lebens aus, in der Simmel nun zu einem doppelten Kompositum greift: "Das Leben findet sein Wesen, seinen Prozeß darin, Mehr-Leben und Mehr-als-Leben zu sein, sein Positiv ist als solcher schon sein Komparativ. $\aleph^{16}$ Denn zum einen sei das Leben stets in Bewegung und erzeuge immer neues Leben, zum anderen bestehe es aber auch im beständigen »Hinausschreiten des Lebens über sich selbst«, insofern »der Tod dem Leben von vornherein einwohnt «. ${ }^{17}$ Das immer grenzüberschreitende Leben wird hier selbst noch mal auf eine Grenze bezogen, der Tod als Gegensatz des Lebens ist

13 Paradigmatisch für diese Vermischung von Werk und Leben gerade bei Goethe ist Gundolf: "Goethes Worte über sein Leben sind selber Formen dieses Lebens, sie treten nicht daraus heraus" (Friedrich Gundolf: Goethe, Berlin [Bondi] 1925, S. 5). Das Leben erscheint hier der gestalthafte Zusammenhang von Leben und Werken, der freilich explizit nur 'Großen zugesprochen wird - ein Konzept, das Benjamin bekanntlich im Wablverwandtschaften-Aufsatz scharf kritisieren wird.

14 Georg Simmel: Der Konflikt der modernen Kultur, in: Gesamtausgabe, Bd. 16, Frankfurt a.M. (Suhrkamp) 1999, S. 181-207, hier S. 183. Expliziert reflektiert Simmel hier auch über die konzeptuelle Undeutlichkeit: "Weil das Leben das Gegenspiel der Form ist, ersichtlich aber nur das irgendwie Geformte mit Begriffen beschreiblich ist, so ist der Ausdruck Leben in dem hier gemeinten, ganz fundamentalen Sinne von einer gewissen Unschärfe, logischen Undeutlichkeit nicht zu befreien" (ebd., S. 205).

15 Simmel: Lebensanschauung, in: Gesamtausgabe, Bd. 16 (Anm. 14), S. 209-425, hier S. 221.

16 Ebd., S. 235.

17 Ebd., S. 229. 
wiederum Teil von diesem - und dieses Gefüge kann nur noch indirekt, durch die verschiedenen Komposita und das für Simmels Schriften so typische dauernde Fortschreiten der Argumentation beschrieben werden. Das Leben kann also gerade deshalb zur universellen Kategorie oder zur absoluten Metapher werden, weil es auf ein Anderes bezogen wird, das aber nie explizit wird, sondern im Text permanent verschoben wird. Wie in anderen Texten wird diese Grenze dabei auch religiös angeschrieben, etwa wenn das "Leben unter dem Schicksalsbegriff" mit dem Glauben an einen lebendigen Gott kontrastiert wird, ${ }^{18}$ oder wenn das Leben als "Kampf in dem absoluten Sinne« bezeichnet wird, »der den relativen Gegensatz von Kampf und Frieden umgreift, während der absolute Frieden, der vielleicht diesen Gegensatz ebenso einschließt, das göttliche Geheimnis bleibt ${ }^{19}{ }^{19}$

Die Nicht-Identität des Lebens mit sich selbst wird in der Folge in mehreren zeitgenössischen Diskursen aufgegriffen und weitergedacht: Die Freud'sche Psychoanalyse wird den Todestrieb einführen und damit die Aporie des $>$ Mehr-alsLebens` im Zusammenspiel zweier Grundkräfte artikulieren. Nicht unähnlich bezieht auch die sich zeitgenössisch entwickelnde Philosophische Anthropologie die Metaphorik des `Lebens` zurück auf ihren Herkunftsbereich, auf das biologische Leben, auf dessen Erforschung sich Max Scheler und Helmut Plessner auf je eigene Art stützten. Diese 'Literalisierung der Metaphorik führt gerade nicht zu einer einfachen `Naturalisierung` des historischen Lebens, sondern im Gegenteil zu einer Differenzierung des Lebensbegriff, insofern bereits in ihm die Spannung einer, mit Plessner gesprochen: `exzentrischen Positionalität entdeckt wird. Gerade durch diesen Ansatz wird dann auch besonders deutlich, dass der biologische Begriff des Lebens auf der Reduktion einer viel komplexeren Semantik beruht, die nicht auf ihn zurückgeführt werden kann.

3.

Für Benjamins frühe Schriften ist 'Leben ein Schlüsselbegriff. Das Leben der Studenten erörtert den Zusammenhang des ,Lebens` mit der Wissenschaft bzw. die Universität als `Lebensform‘; der Hölderlin-Kommentar führt das `Gedichteter explizit als Grenzbegriff zwischen dem Gedicht und dem `Leben "Fortleben« spricht Benjamin, um die "Zeugnisse» eines Menschen (wie Briefe) von seinen Werken zu unterscheiden: „Die `Zeugnisse` gehören zur Geschichte

18 Ebd., S. 307.

19 Simmel: Der Konflikt der modernen Kultur (Anm. 14), S. 207. Charakteristischerweise schließt der Text mit dieser Formulierung, weil eine Universalisierung der Idee des Konfliktes eben nur in diesem Gegensatz ein Halten findet. 
des Fortlebens eines Menschen und eben, wie in das Leben das Fortleben mit seiner eigenen Geschichte hereinragt, läßt sich am Briefwechsel studieren. (Nicht so an den Werken, in ihnen vermischen sich nicht Leben und Fortleben, sondern die Werke sind wie eine Wasserscheide.)«(GS VI, 95) Dass sich Benjamin vermittelt über seinen Lehrer Paul Häberlin auch mit der anthropologischen Frage der Beziehung von Leib und Körper befasste, zeigen seine Aufzeichnungen zum psychophysischen Parallelismus, die - nicht unähnlich wie bei Simmel - das Leben in Beziehung zur Vergänglichkeit einerseits, zur Unsterblichkeit andererseits setzen. ${ }^{20}$

Es ist aber vor allem der Übersetzeraufsatz, in welchem der Lebensbegriff eine zentrale und bisher gegenüber den Konzepten der rreinen Spraches, der ,Wörtlichkeit‘ und der 'Aufgabe des Übersetzens weitgehend vernachlässigte Rolle spielt. ${ }^{21}$ Benjamin versteht die Übersetzung bekanntlich von der Übersetzbarkeit als Bedingung der Möglichkeit von Dichtung: ,Übersetzung ist - wie vorher 'Kritik und später 'Reproduktion - nicht etwas sekundär und äußerlich dem übersetzten Text Hinzugefügtes, sondern gründet in diesem selbst. Es ist, so mag man paraphrasieren, für Texte wesentlich, übersetzt zu werden, denn das gehört zu ihrer Geschichte, und Texte sind wesentlich geschichtlich.

Gerade diese Geschichtlichkeit wird aber im Aufsatz mit der Kategorie des Lebens` eingeführt, welche mithin eine zentrale Stelle im Text hat. Nachdem Benjamin zuerst apodiktisch Missverständnisse abgewehrt hat und seinen Begriff der `Form` setzend eingeführt hat, bestimmt er den als wesentlich behaupteten Zusammenhang von Original und Übersetzung als "Zusammenhang des Lebens«: "So wie die Äußerungen des Lebens innigst mit dem Lebendigen zusammenhängen, ohne ihm etwas zu bedeuten, geht die Übersetzung aus dem Original hervor" (GS IV, 10). Der folgende Absatz führt diese Lebensbeziehung aus und gibt ihr eine grundsätzliche Bedeutung: »In völlig unmetaphorischer Sachlichkeit ist der Gedanke vom Leben und Fortleben der Kunstwerke zu erfassen.» (11) Denn 'Leben sei eben nicht nur dem Organischen eigen, sondern

20 Vgl. dazu Uwe Steiner »Von Bern nach Muri. Vier unveröffentlichte Briefe Walter Benjamins an Paul Häberlin im Kontext«, in: DVJS 77 (2001), S. 463-490. Generell zu Benjamins Lebensbegriff einer Kreatürlichkeit in Doppelreferenz zur leiblichen Basis des Lebens einerseits und zur Geschöpflichkeit andererseits vgl. Sigrid Weigel: Walter Benjamin: Die Kreatur, das Heilige, die Bilder, Frankfurt a. M. (Fischer) 2008.

21 Heinrich Kaulen berührt das Konzept nur kurz und konstatiert dass Benjamins Konzeption »nicht mit dem Lebensbegriff der Lebensphilosophie zu verwechseln« sei, ohne das näher zu untersuchen (Rettung und Destruktion. Untersuchungen zur Hermeneutik Walter Benjamins, Berlin (De Gruyter) 1987, S. 39. Werner Hamacher interpretiert das Überleben als "Überleben im Zitat«, dass den Text erst eigentlich sprachlich mache: „Erst in ihrem ,Überleben spricht die Sprache sich selbst und nicht mehr von etwas und jemanden“ ("Intensive Sprachen«, in: Christiaan L./Hart Nibbrig: Übersetzen: Walter Benjamin, Frankfurt a.M. [Suhrkamp] 2001, S. 174-235, hier S. 184). 
vielmehr in allem, "wovon es Geschichte gibt und was nicht allein ihr Schauplatz ist" (ebd.). Diese wesentliche und nicht nur äußerliche Geschichtlichkeit soll also als Lebens-Geschichte beschrieben werden, und die sunmetaphorischer Sachlichkeit dieser Vorstellung soll offensichtlich durch eine Analogisierung der kulturellen Zeit mit Phänomenen des Lebens plausibilisiert werden: „Die Geschichte der großen Kunstwerke kennt ihre Deszendenz aus den Quellen, ihre Gestaltung im Zeitalter des Künstlers und die Periode ihres grundsätzlich ewigen Fortlebens bei den nachfolgenden Generationen« (ebd.). Zu diesem Fortleben gehört nun auch die Übersetzung, bei deren weiterer Bestimmung die Idee des Lebens später wieder aufgegriffen wird, wenn es heißt, in der Übersetzung erreiche »das Leben des Originals seine stets erneute späteste und umfassendste Entfaltung« (ebd.), vor allem aber, wenn die Äquivalenz von Original und Übersetzung als Kriterium zurückgewiesen wird, weil »Ähnlichkeit nicht notwendig bei Verwandtschaft sich einfinden muß« (13). ${ }^{22}$ Schließlich ändere sich mit der historischen Situation eines Textes auch seine Bedeutung: „Denn in seinem Fortleben, das so nicht heißen dürfte, wenn es nicht Wandlung und Erneuerung des Lebendigen wäre, ändert sich das Original. Es gibt eine Nachreife auch der festgelegten Worte« (12). Das Kunstwerk lebt also, weil es eine Abstammung hat, weil es gestaltet ist und weil es nachreifen wird.

Benjamins Überlegungen greifen offensichtlich auf seine Beschäftigung mit der deutschen Frühromantik zurück, die, wie Benjamin betont »vor anderen Einsicht in das Leben der Werke besessen« habe (15). Wie die Romantiker will Benjamin das Verhältnis der Kunstwerke zur Kunst als ein mediales Verhältnis beschreiben, das wie bei Dilthey als "Zusammenhang des Lebens" aufgefasst wird. ${ }^{23}$ Dieser Zusammenhang ist `ewig`, das heißt er stiftet Kontinuität, aber diese Ewigkeit verweist zugleich auf einen anderen Diskurszusammenhang, von dem aus deutlich wird, dass Benjamins Interessen in eine andere Richtung gehen als die Diltheys. Das rewige Fortleben »heißt, wo es zutage tritt, Ruhm. Übersetzungen, die mehr als Vermittlungen sind, entstehen, wenn im Fortleben ein Werk das Zeitalter seines Ruhmes erreicht hat. [...] In ihnen erreicht das Leben des Originals seine stets erneute späteste und umfassendste Entfaltung" (11). Das Fortleben eines Werkes betrifft also seinen `Ruhm`, und wenn Benjamin noch im Passagenwerk die Analyse des `Nachlebens` mit der seines `Ruhmes gleichsetzt (GS V, 574 f.) - was im Kontext seines Interesses an der Tradition

22 In diesem Sinne wurde Übersetzung schon in Über die Sprache überhaupt von einer bloßen Äquivalenz-Relation abgesetzt: "Die Übersetzung ist die Überführung der einen Sprache in die andere durch ein Kontinuum von Verwandlungen. Kontinua der Verwandlung, nicht abstrakte Gleichheits- und Ähnlichkeitsbezirke durchmißt die Übersetzung« (GS II, 151).

23 Zur Bedeutung der frühromantischen Theorie der Kritik für den Übersetzeraufsatz vgl. insb. Uwe Steiner: Die Geburt der Kritik aus dem Geiste der Kunst, Würzburg (Könighausen \& Neumann) 1989. 
der Unterdrückten eher verwundert -, zeigt das, wie wichtig dieser Diskurs für ihn ist. Nun gehört es zum Bestand der Tradition des literarischen Ruhms, dass der Ruhm des Dichters, der ihn unsterblich macht, nach seinem Tod entsteht. ${ }^{24}$ Dabei handelt es sich nicht nur um das Phänomen der verspäteten Rezeption, sondern Ruhm und Tod stehen hier in einem intrinsischen Zusammenhang: Die poetische ,Unsterblichkeit ist nicht einfach eine Fortdauer, sondern Fortdauer im Tod, fast durch den Tod, sie korreliert jedenfalls mit dem körperlichen Verschwinden des Poeten. Bei Horaz, wohl einer der Urszenen des poetischen Ruhmes, heißt es "non omnis moriar multaque pars mei / vitabit Libitinam» (»Sterben werde ich nicht ganz, viel von mir wird einst der Todesgöttin entfliehen«). ${ }^{25}$ Das Monument, das sich der Dichter mit seinem Werk errichtet, ist zugleich auch ein Kenotaph, ein Grabmal, der Ruhm selbst in seiner Ewigkeit wird immer zusammen gedacht mit der Vergänglichkeit des Menschen und der ewigen Vergängnis aller Dinge. Es ist dieser Aspekt des `Nachlebens`, der bestimmend für Benjamins Begriff ist.

\section{4.}

Das Fortleben des Kunstwerkes ist daher für Benjamin nicht einfach seine Fortdauer. Im Text drückt sich das durch eine Verschiebung aus, die Benjamin bei der Einführung des Ausdrucks vornimmt:

Daß eine Übersetzung niemals, so gut sie auch sei, etwas für das Original zu bedeuten vermag, leuchtet ein. Dennoch steht sie mit diesem kraft seiner Übersetzbarkeit im nächsten Zusammenhang. Ja, dieser Zusammenhang ist um so inniger, als er für das Original selbst nichts mehr bedeutet. Er darf ein natürlicher genannt werden und zwar genauer ein Zusammenhang des Lebens. So wie die Äußerungen des Lebens innigst mit dem Lebendigen zusammenhängen, ohne ihm etwas zu bedeuten, geht die Übersetzung aus dem Original hervor. Zwar nicht aus seinem Leben so sehr denn aus seinem ,Überleben`. Ist doch die Übersetzung später als das Original und bezeichnet sie doch bei den bedeutenden Werken, die da ihre erwählten Übersetzer niemals im Zeitalter ihrer Entstehung finden, das Stadium ihres Fortlebens. (GS IV 10 f.)

24 Vgl. dazu Detlev Schöttker: „Ruhm und Rezeption. Unsterblichkeit als Voraussetzung der Literaturwissenschaft«, in: Jörg Schönert (Hg.): Literaturwissenschaft und Wissenschaftsforschung, Stuttgart u. a. (Metzler) 2000, S. 472-487 sowie Konrad Paul Liessmann: Ruhm Tod und Unsterblichkeit, Wien (Zsolnay) 2004.

25 Horaz: Carmen 3,30: An Melpomene. 
Benjamins Übergang vom `Leben` zum `Überleben` hat wesentliche Folgen für die Argumentation, weil erst durch ihn das Original von der Übersetzung unterschieden wird: Diese mag zwar aus jenem hervorgehen, aber sie geht nicht in es zurück, in ihr mag seine Bedeutung fortleben und nachreifen, nicht aber sein Wortlaut. Erst dieser Übergang unterscheidet Benjamins `Nachleben` von anderen Konzeptionen der Fortdauer, der Wirkungsgeschichte, aber auch der Nachträglichkeit. Aus dem Nachleben im Sinne der Kontinutiät wird ein Nachleben im Sinne des ,Überlebens`, was eben ein Leben nach dem Leben des Originals ist. Die Rede vom `Nachleben Lebensbegriff, dem der Tod das andere ist - denn dann ist das Nach-Leben eben kein Leben mehr, sondern der Tod -, als auch für jenen, dem der Tod Teil des Lebens ist - denn dann gibt es kein Nach-Leben mehr, weil auch der Tod noch zum Leben gehört. Vom 'Nachleben` lässt sich daher begrifflich nicht sprechen, sondern nur mittels einer Figur, die hier nicht einfach eine Metapher ist, sondern eine komplexere textuelle Operation. ${ }^{26}$ Komplex ist wie gezeigt schon der semantische Hintergrund des 'Lebens», indem ein Mehr-Leben immer schon mitgedacht ist. Für Benjamin ist aber darüber hinaus entscheidend, dass diese Semantik zugleich »in völlig unmetaphorischer Sachlichkeit« literalisiert und verschoben wird, vor allem aber, dass diese beiden Bewegungen selbst durch den Vollzug des Textes in Beziehung zueinander gesetzt werden. Gerade damit wird dann auch die Semantik vom Leben aufgenommen und überschrieben, aber nicht ersetzt oder gar aufgehoben.

Der Text markiert diese Bewegung auf mehrfache Weise: Erstens durch den anakoluthischen Satz, in dem sie vollzogen wird, in dem nichts geschieht, als dass das Wort `Leben` durch ,Überleben` ersetzt wird. Er lässt in seiner Fügung offen, ob das ,Überleben` hier nur ein anderer Name für das `Leben` ist oder sich von ihm unterscheidet bzw. wo denn die Grenze zwischen dem `Leben kes und seinem `Überleben` zu ziehen wäre: im Moment der Niederschrift, beim Druck, in der Fassung letzter Hand, beim Tod des Autors oder gar beim Sprachwechsel, der mit der Übersetzung einhergeht? Zweitens erscheint das Ziel dieser Verschiebung, das ,Überleben` in Anführungszeichen. Während das Leben (und auch das Fortleben) der Werke in "völlig unmetaphorischer" Weise gemeint sein soll, erscheint das Überleben anders, weniger wörtlich gemeint. Auch das lässt verschiedene Lektüren offen: etwa als idiosynkratische Wendung, so dass ,Überleben` im Sinne nicht des üblichen Überlebens gemeint ist, sondern vielleicht im Sinne von Über-Leben, parallel zu Simmels Mehr-Leben. Oder man kann die Anführungszeichen als Markierung eines metaphorischen Sprachgebrauchs

26 Überleben sei "not simply as that which comes rafter life has gone, but a life that is rafter itself - that is, constantly in pursuit of what it will never be« (Weber: "Translatability» [Anm. 4], S. 66). 
verstehen, was die verwirrenden Konsequenz hätte, dass die Werke wirklich leben` würden aber nur metaphorisch ‘überleben` würden, während es doch der unbefangenen Ansicht eher umgekehrt erscheint. Die Anführung gibt dem ,Überleben` also eine zusätzliche Ambiguität, die den Ausdruck von einer einfachen Metapher zu einer Figur macht, die sich nicht mehr auf ein wirklich Gemeintes reduzieren lässt. ${ }^{27}$ Drittens schließlich wird das ,Überleben`sogleich durch einen weiteren Terminus ergänzt, durch das 'Fortleben`, das auch die Konnotationen der Entfernung und Entäußerung aufruft, »living on, but also living away«. ${ }^{28}$ Wieder ist die semantische Beziehung unklar - ist es synonym mit ,Überleben` oder unterscheidet sich ihre Bedeutung, wie verhält es sich seinerseits zum 'Leben - so dass sich die metonymische Kette von 'Leben zu ,Überleben ‘fortsetzt und auf diese Weise verhindert, dass man ,Überleben', 'Fortleben` und 'Nachleben` in ihrer Bedeutung differenzieren und damit wenigstens im Ansatz definieren könnte oder das sich die Denkfigur des Textes in einem `Begriff^ des Überlebens beruhigen könnte.

\section{5.}

`Nachleben bedeutet für Benjamin also zugleich mehr und weniger als das bloße Fortbestehen: Weniger, weil das Nachleben eben nur ein ,Überleben ist, gewissermaßen eine Diminutivform des Lebens, die noch dazu in Anführungszeichen zu setzen ist; mehr, weil es ein "grundsätzlich ewiges Fortleben« betrifft, weil es um `Unsterblichkeit` des Ruhms geht. Nun ist ein solches Leben nach dem Leben, sei es vermindert oder vermehrt, kaum ohne religiöse Semantik denkbar, sei es die einer christlichen Unsterblichkeit oder einer paganen Gespensterfurcht. Benjamins `Nachleben` ist demnach immer auch `Nachleben der Religion` und betrifft damit einen zentralen und bis heute umstrittenen und problematischen Bereich von Benjamins Werk: seinen expliziten Rekurs auf religiöse Praktiken und theologische Denkfiguren. Dabei kann man die Figur des 'Nachlebens doppelt lesen, je nachdem, wie man den Genitiv in 'Nachleben der Religionen versteht: Einerseits denkt Benjamin im `Nachleben` die historische Überlieferung unter Zuhilfenahme religiöser Modelle und Semantiken, andererseits liest er die Geschichte dieser Semantik selbst als deren `Nachleben`, als grundsätzlich

27 Vgl. dazu Hamacher: „Überleben - und so auch Übersetzung - ist jeweils ein Überleben in Anführungszeichen, es ist, wie Benjamin schreibt, Überleben', weil es nicht nur ein Leben über das Leben hinaus, nicht nur ein Leben nach dem Leben [...] ist, sondern weil dieses Überleben im Wort - im Wort, Überleben` zum Beispiel - nur ein Überleben im Zitat, also kein Überleben, das noch Überleben bedeutete, vielmehr ein nicht mehr bedeutendes, sondern sprachlich [...] verwirklichtes ist« (Hamacher: »Intensive Sprachen« [Anm. 21], S. 184).

28 Weber: »Translatability« (Anm. 4), S. 67. 
ewige, aber zugleich gespenstische Nachgeschichte ursprünglich religiöser Ideen im Raum der profanen Kultur.

Dass Benjamins Überlegungen zur Geschichtlichkeit des kulturellen `Lebens` auf religiöse Semantik rekurriert, zeigt sich nicht erst, wenn von der 'Unsterblichkeit von Kunstwerken die Rede ist, sondern bereits in der Idee von 'Tradition oder von `Lehre`, die vor allem in den frühen Aufzeichnungen zentral sind, aber auch später immer wieder auftauchen. Beides sind keine abstrakten Begriffe. In einem Brief an Scholem vom September 1917 beschreibt Benjamin »die Tradition" zunächst als "das Medium in dem sich kontinuierlich der Lernende in den Lehrenden verwandelt«: „Wer sein Wissen als überliefertes begriffen hat in dem allein wird es überlieferbar« (GB I, 382). ${ }^{29}$ Indem die Kontinuität der Erfahrung hier zugleich logisch und zeitlich gedacht wird, reformuliert Benjamin damit die für den Sprachaufsatz zentrale Kategorie der Mitteilbarkeit als TTradierbarkeit`, aus der dann später auch `Kritisierbarkeit` und „Übersetzbarkeit entwickelt werden können. Benjamin macht hier aber auch deutlich, es sei »schwer« über diese Phänomene zu reden, "weil deren Ordnung mit der Religiösen Ordnung der Tradition ganz zusammen fällt« (382 f.). Offensichtlich stehen seine Überlegungen in engem Zusammenhang mit Scholems gleichzeitig unternommenen umfassenden Versuchen, die jüdische Tradition philosophisch zu beschreiben, deren Relevanz für Benjamins frühes Denken noch nicht annähernd berücksichtigt ist. Das ist auch deshalb nicht geschehen, weil Scholems Überlegungen und generell das 'Jüdischer bei Benjamin meist mit mehr oder weniger deutlicher Abwehrhaltung oder Faszination, jedenfalls mit Alterisierungsgestus als ımystisch oder `messianisch charakterisiert worden sind. Dagegen lässt sich gerade an den Aufzeichnungen Scholems zeigen, dass es nicht um Glaubenssätze oder spekulative Metaphysik geht, sondern um den - allerdings oft spekulativen - Versuch, bestimmte Erfahrungen und Phänomene der jüdischen Traditionsliteratur zu beschreiben, etwa den Zusammenhang von mündlicher und schriftlicher Überlieferung oder auch Probleme der Übersetzung, also durchaus materiale Überlieferungsphänomene. ${ }^{30}$ Schon bei Scholem kann man deutlich erkennen, dass dieser Versuch einer >Philosophie des Judentums` immer wieder auf problematische und auch paradoxe Formulierungen, Aphorismen und Allegorien hinausläuft. ${ }^{31}$ Ähnlich finden auch Benjamins Überlegungen zum `Medium`der Tradition im Brief an Scholem keinen begrifflichen Abschluss, sondern kulminieren in einem

29 Zeitgleich entwirft Benjamin als Programm der kommenden Philosophie die Aufgabe, "einen Erkenntnisbegriff zu schaffen dem der Begriff einer Erfahrung korrespondiert von der die Erkenntnis Lehre ist« (GS II, 168).

30 Vgl. dazu meine Dissertation: Gershom Scholem. Politisches, esoterisches und historiographisches Schreiben, München (Fink) 2002, insb. Kap. 2.

31 Vgl. zu diesen Schreibverfahren auch meinen Aufsatz »Reading Gershom Scholem», in: Jewish Quarterly Review 96 (2006) 2, S. 203-231. 
Bild: „Die Lehre ist wie ein wogendes Meer, für die Welle aber (wenn wir sie als Bild des Menschen nehmen) kommt alles darauf an sich seiner Bewegung so hinzugeben, daß sie bis zum Kamm wächst und überstürzt mit Schäumen.» (Ebd.) In dieser Figur findet sich nicht nur das Moment der Kontinuität, eines kontinuierlichen Wellenschlages der Tradition, sondern auch die Idee der Unterbrechung, des Traditionsbruches, welche im Brechen der Wellen geradezu wörtlich Gestalt annimmt.

Die `Lehre und die ihr eigene Geschichtlichkeit spielen auch im Übersetzeraufsatz eine zentrale Rolle. Bekanntlich schließt der Text mit einem Rekurs auf die heiligen Texte, die scheinbar unmittelbar in der reinen Sprache aufgehen: "Wo der Text unmittelbar, ohne vermittelnden Sinn, in seiner Wörtlichkeit der wahren Sprache, der Wahrheit oder der Lehre angehört, ist er übersetzbar schlechthin." (GS IV, 21) Wenn Benjamins Erörterungen zunächst apodiktisch mit dem Postulat der Übersetzbarkeit begannen, so wird nun die schlechthinnige Übersetzbarkeit als Wesen der Lehre bezeichnet. So scheint der Text mit einer Schließung zu enden, die dann, ebenso wie die Idee der reinen Sprache, oft als ımessianischer Figur gelesen worden ist, mit welcher der Text sich vor der Konsequenz dekonstruktiver Logik schütze. ${ }^{32}$ Es ist aber fraglich, ob Benjamin hier wirklich stheologisch oder ımystisch argumentiert und nicht vielmehr historisch an den heiligen Texten eine besondere Form des Nachlebens beobachtet: 'Heilig` wären Texte nicht aufgrund bestimmter inhaltlicher oder formaler Merkmale, sondern aufgrund ihrer Funktion: weil sie slebendig, so überliefert werden, dass Kommentierung (und Übersetzung) immer wieder aufihr Original zurückkommen. ${ }^{33}$ Heilig wären mit anderen Worten Texte, deren Nachleben nicht von ihrem Leben unterschieden werden, weil sie nicht sterben`, weil etwa ihre Autorintention niemals relevant gewesen ist oder von ihnen im Laufe der Kanonisierung abgefallen ist - genau das ist bei der biblischen Überlieferung der Fall. An anderer Stelle hat Benjamin auch für dieses Leben ein ingeniöses Bild gefunden: "Kommentar und Übersetzung verhalten sich zum Text wie Stil und Mimesis zur Natur: dasselbe Phänomen unter verschiedenen Betrachtungsweisen. Am Baum des heiligen Textes sind beide nur die ewig rauschenden Blätter, am Baume des profanen die rechtzeitig fallenden Früchte." (GS IV, 92) Das Bild beschreibt

32 Kaulen spricht etwa vom »theologischen Modell der Schriftübertragung« (Rettung und Destruktion [Anm. 21], S. 75), durch das Benjamins Text "auf den ersten Blick« in "positive Theologie« münden würde (ebd., S. 84), wenn das nicht durch Rekurs auf die >deutscher Tradition der wörtlichen Übersetzung (Hölderlin) verhindert werde. Nach Irving Wohlfahrt schließt sich hier der Kreis: „Die Bibel stellt das Faktum der Offenbarung dar" (vgl. „Das Medium der Übersetzung« in: Hart Nibbrig: Übersetzen: Walter Benjamin, S. 80-130, hier 117), obwohl hier nicht von der Bibel, sondern von deren Übersetzung die Rede ist.

33 Vgl. zu dieser Theorie einer skripturalen 'Funktion` (die durchaus im Sinne Jakobsons zu interpretieren wäre) Brian Britt: Walter Benjamin and The Bible, New York (Continuum International Publishing) 1996, insb. S. 51-69. 
sehr präzise das `Leben` des heiligen Textes, in dem Kommentar und Übersetzung das Original nicht ablösen, sondern immer schon mit ihm zusammengehören, jedenfalls solange der Text noch `lebendig` ist.

Dass der Heilige Text seine Übersetzbarkeit weniger impliziert als voraussetzt, und dass er für Benjamins Text nicht die Reinheit jenseits der Übersetzung figuriert, sondern vielmehr die Übersetzung selbst, macht vor allem der Schluss des Übersetzer-Aufsatzes deutlich: „Die Interlinearversion des heiligen Textes ist das Urbild oder Ideal aller Übersetzung." (GS IV, 21) Der Text schließt also gar nicht mit dem heiligen Text, sondern mit dessen Interlinearversion, die gerade die $\mathrm{Zu}-$ sammengehörigkeit von Original und Übersetzung präzise ausdrückt, welche die funktionale Heiligkeit des Textes ausmacht. Eine Interlinearübersetzung ist dabei immer ein komplexes Gefüge, welche keine utopische Einheit der Sprache figuriert, sondern immer eine Agentur der Differenz ist. Und zwar ist das ganz besonders der Fall, wenn wir annehmen, dass Benjamin hier an eine Interlinearversion der hebräischen Bibel denkt. Denn eine solche ist nur schwer lesbar`, weil man aufgrund der umgekehrten Laufrichtung der hebräischen Schrift in deren deutscher Interlinearübersetzung die Zeile von rechts nach links, das Wort aber von links nach rechts lesen muss. Die Interlinearversion figuriert daher an dieser Stelle selbst allenfalls eine umwegige und komplizierte Lesebewegung, die gewissermaßen in Epizyklen fortschreitet; es handelt sich um eine fast unlesbare Schrift, die auf Lesbarkeit nur deutet - auf die Lesbarkeit des Hebräischen nämlich, aus welcher derjenige, der sich die Interlinearversion ansieht - sagen wir: Benjamin -, ausgeschlossen ist.

6.

Benjamins Figuren der Interlinearversion oder der brechenden Welle der Tradition markieren eine Grenze. Ob eine unlesbare Schrift noch Schrift sei und nicht vielmehr "Leben«, ob die Tradition mit ihrem Abbruch verschwindet oder in ihren "Wellentälern« nur latent werde, diskutiert Benjamin später in dem Briefwechsel, den er mit Gershom Scholem über Kafka führt. ${ }^{34}$ Auch diese Dimension der Unentscheidbarkeit ist zentral für Benjamins Figur des `Nachlebens`, auch sie taucht immer wieder im Verlauf des Übersetzeraufsatzes auf, ohne dass das hier erschöpfend thematisiert werden könnte. Die Übersetzung etwa wird als Wachstum` und `Entfaltung beschrieben, das aber Züge eines hybriden ,ÜberWachstums hat, denn in der Übersetzung "wächst das Original in einen gleichsam höheren und reineren Luftkreis der Sprache hinauf, in welchem es freilich

34 Vgl. Walter Benjamin, Gershom Scholem, Briefwechsel, Frankfurt a.M. (Suhrkamp) 1995, insb. S. 167 u. 286. Scholem schreibt hier irrtümlich von "Wellenbergen« - er mag nur das Bild erinnern und dessen Bezug vergessen haben. 
nicht auf die Dauer zu leben vermag" (GS IV, 14). Während im Original ein 'natürliches` Verhältnis von Gehalt und Sprache herrscht - sie bilden »eine gewisse Einheit wie Frucht und Schale« -, so ist das in der Übersetzung zumindest gelockert, hier "umgibt die Sprache der Übersetzung ihren Gehalt wie ein Königsmantel in weiten Falten« (15), bzw. hier wird das Original in einen höheren Sprachbereich »verpflanzt» (ebd.). 'Heraufwachsen und 'Verpflanzen` sind hier zwei reziproke Bewegungen, wie auch die Figur der Nachreife deuten sie bereits an, dass das steigernde ü̈ber der Übersetzung oder Überlieferung nicht nur eine Fortsetzung, sondern auch eine Verflüchtigung und eine Auflösung bedeuten kann - vom Boden gelöst, wächst die Übersetzung in der Luft.

Damit stellen sich aber auch die Beziehung des 'Nachlebens` zu 'Tradition', 'Lehre und insgesamt zur religiösen Semantik noch komplexer dar als auf den ersten Blick erschien. Die Debatte mit Scholem kreist immer auch um die Frage, ob die brechende Tradition und die unlesbare Schrift 'noch religiös sind, denn wie erwähnt, impliziert Benjamins `Nachleben` nicht nur eine religiöse Semantik, sondern denkt diese selbst im Prozess der Verschiebung und Verwandlung. Auch die religiöse Semantik ist dementsprechend kein fester Grund der Argumentation, sondern befindet sich in permanenter Auflösung, die zentral für die Bewegung von Benjamins Texten ist. Das `Nachleben` führt gewissermaßen selbst nur ein Nachleben, und aus seiner Potenzierbarkeit entspringen die Energien, welche die Figur so stark und prägnant machen.

Die ambige Erscheinungsform des Religiösen ist dabei nicht unbedingt spezifisch für Benjamin, sondern charakterisiert die zeitgenössische Debatte über 'Säkularisierung wie man sie insbesondere bei Max Weber und seinen Nachfolgern wie Carl Schmitt, Karl Löwith oder Friedrich Gogarten findet. Auch deren Diskurs basierte nicht nur auf der metaphorischen Übertragung der Säkularisierung als Aneignung von Kirchengütern auf einen geistesgeschichtlichen Prozess, sondern wesentlich auf der Verschränkung einer metaphorischen Ähnlichkeit („Der Kapitalismus ist wie Religion`) mit einer genealogischen Beziehung (`Der Kapitalismus war Religion`), die stets zugleich Identität und Differenz von Religion und Moderne behauptete. ${ }^{35}$ So ist bei Weber der `Geist` des Kapitalismus zwar religiös gedacht, aber zugleich im Verschwinden begriffen, weil die ,Wurzek der innerweltlichen Askese im entwickelten Kapitalismus am Absterben sei. Bei Schmitt wird die ,Übertragung theologischer Konzepte auf die Politik selbst programmatisch, wobei sich unter der Hand allerdings die Richtung der Übertragung umkehrt, insofern er zwar explizit einen historischen Übertragungsprozess von der Theologie zur Politik behauptet (den er dann allerdings kaum untersucht), implizit aber eher die eigene politische Begrifflichkeit theologisch auflädt. Gogarten schließlich denkt den historischen Prozess der Säkularisierung in der Neuzeit selbst noch einmal theologisch als Trennung von

35 Vgl. Daniel Weidner: »Rhetorik der Säkularisierung«, in: DVJs 78 (2004) 1, S. 95-132. 
Göttlichem und Weltlichem und literarisiert damit die Metapher einer historischen Verweltlichung theologisch. Alle diese Entwürfe tragen zu einem hochgradig überdeterminierten Diskurs von Säkularisierung bei, der für die Debatten der Zwischenkriegszeit konstitutiv ist.

Benjamins Texte gehören offensichtlich in dieses Diskursfeld, sind dort aber schwer zu verorten. ${ }^{36}$ Sie lassen sich wohl auch weniger als Positionierungen auf der einen oder anderen Seite verstehen denn als Versuch, in diesem Diskursfeld zu denken und zu schreiben: So wenig wie die anderen erwähnten Autoren hat Benjamin eine `Theorie‘ der Säkularisierung, stärker als sie ist er sich der Ambivalenzen und Ambiguitäten dieses Diskurses bewusst und versucht, diese in seinen Denkfiguren darstellbar zu machen. Wenn daher der Kategorie der Säkularisierung nicht zu Unrecht Substanzialismus vorgeworfen worden ist - sie suggeriere, so Hans Blumenbergs nach wie vor zentrale Kritik, eine gleichbleibende religiöse Substanz in allen Verwandlung -, so kann Benjamins Figur des `Nachlebens ein Korrektiv sein. Sie unterläuft lineare Narrative und klare Oppositionen und legt dabei auch die Figürlichkeit von Säkularisierung wieder frei, die sich in der substanzialistischen Verhärtung ebenso verloren hat wie in der Umsetzung in ein historisches Forschungsprogramm durch andere, vermeintlich trennschärfere und neutralere Begriffe. Für Benjamin - und das ist das Aufregende und Aktuelle seiner Theologie - lassen sich religiöse Praktiken und Symboliken eben nicht diskursiv auf den Begriff bringen oder historisierend festlegen, sondern halten als Erinnerte das Denken in Bewegung und müssen vom Denken in Bewegung gehalten werden, gerade wenn es um Überlieferung und Geschichte geht: »im Eingedenken machen wir eine Erfahrung, die uns verbietet, die Geschichte grundsätzlich atheologisch zu begreifen, so wenig wir sie in unmittelbar theologischen Begriffen zu schreiben versuchen dürfen« (GS V 589).

7.

Benjamins Texte sind nicht eigentlich begrifflich aufgebaut, sondern kreisen um Figuren: um zentrale Metaphern, um terminologische Verschiebungen oder Begriffskonstellationen. Diese Figuren arbeiten in den Texten: Sie entfalten

36 Vgl. als Bestandsaufnahme der kontroversen Debatte Uwe Steiner: „Säkularisierung. Überlegungen zum Ursprung und zu einigen Implikationen des Begriffs bei Benjamin", in: ders. (Hg.): Walter Benjamin 1892-1940, Bern (Peter Lang) 1992, S. 139-187. Zur Unterscheidung von Benjamins "Doppelreferenz" auf sakrales und natürliches von einer Anverwandlung theologischer Semantik vgl. Sigrid Weigel: „Während die politische Theologie die theo-logischen Begriffe beerbt, geht es in Benjamins Denken um Phänomene und Bedeutungen, in denen verschwundene religiöse und kultische Praktiken in der Moderne fortleben « (dies.: Walter Benjamin [Anm. 20], S. 22). 
sich im Verlauf der Argumentation, bestimmen deren Semantik untergründig und schreiben zentrale Probleme des zeitgenössischen Diskurses in sie ein. Oft werden sie auch als solche markiert, wie im Falle des `Nachlebens`, das schon als Wort - ein Leben nach dem Leben -, vor allem aber in der Weise seiner Einführung und textuellen Entwicklung als Figur erkennbar ist: Der Text sperrt sich gewissermaßen selbst gegen eine literale Lektüre und zwingt den Leser zu Umwegen. Solche Denkfiguren sind nicht so feste Verbindungen wie Begriffe, Konzepte oder Ideen, sondern haben etwas Vorläufiges und Vorsichtiges - aber gerade darin ist es ihnen möglich, Grenzen zu überschreiten und sie in dieser Überschreitung auch aufzuzeigen. Sie können neue Anschlüsse produzieren, den Dingen einen Ort im Diskurs geben und die Paradoxien der kulturellen Semantik lesbar machen, die sie verarbeiten und verdichten.

Im Fall des `Nachlebens` lässt sich beobachten, wie Benjamin eine solche Figur entwickelt, die auf einen allgemeinen kulturpolitischen Ausdruck und auf einen philosophischen Diskurs rekurriert, beide aber entscheidend modifiziert, um Geschichte und deren Untersuchung neu zu denken. Eine umfassendere Lektüre hätte die von der Forschung bisher eher vernachlässigte Beziehung Benjamins zu Lebensphilosophie und Anthropologie genauer ins Auge fassen und zugleich nachzeichnen müssen, wie sich die Semantik des `Lebens` in Benjamins späteren Texten verschiebt. Denn die explizite Rede vom `Nachleben scheint dort in den Hintergrund zu treten - man kann vermuten, dass seine 'materialistischer Umorientierung zu einer wachsenden Reserve gegenüber dem Wortfeld des `Lebens` führt und dass die gedächtnistheoretische Umarbeitung sein Interesse in andere Bahnen gelenkt hat. Die Impulse der Denkfigur sind aber nicht nur zentral für Benjamins andauernde Beschäftigung mit Überlieferungs- und Rezeptionsprozessen in seiner Arbeit als Literaturkritiker, Historiker und Geschichtstheoretiker, sondern finden sich auch in diversen Figuren des unheimlich heilig-gespenstischen 'Lebens in seinen kritischen, politischen und autobiographischen Schriften. Benjamins Nachdenken über diese weite, keineswegs biologistische Idee des ‘Lebens` und 'Nachlebens` könnte gerade heute von zentraler Bedeutung sein, wo die Kulturwissenschaft sich auf ihre eigenen historischen und epistemologischen Grundlagen besinnt und zugleich der `Lebenswissenschaft als einem neuen Paradigma des Wissens begegnet. 